\title{
High-performance freestanding thermoelectric membrane by intact exfoliation of van der Waals epitaxial bismuth antimony telluride film
}

Liangwei Fu

Sungkyunkwan University

Kwansu Park

Sungkyunkwan University

Kyu Hyoung Lee

Yonsei University https://orcid.org/0000-0001-6843-6706

Bongju Kim

Seoul National University

Wooseon Choi

Sungkyunkwan University

Young-Min Kim

Sungkyunkwan University

\section{Sang-il Kim}

University of Seoul https://orcid.org/0000-0003-0093-720X

Jae-Yeol Hwang

Pukyong National University https://orcid.org/0000-0002-9796-4329

Sung Wng Kim ( $\square$ kimsungwng@skku.edu )

Sungkyunkwan University

\section{Article}

Keywords: Functional Heterostructures, Sapphire Substrate, Epitaxy, Pseudomorphic Te Monolayer, Highquality Crystallinity, Arbitrary Shaped Thermoelectric Devices

Posted Date: November 16th, 2020

DOI: https://doi.org/10.21203/rs.3.rs-103826/v1

License: (1) (1) This work is licensed under a Creative Commons Attribution 4.0 International License.

Read Full License 


\section{Abstract}

Separation of epitaxial thin films on growth substrate and transferring onto other materials for functional heterostructures have boosted the transformative impact on science and technology. However, this scheme has proved challenging in thin film thermoelectrics, but promised a vast range of applications beyond the limited device configurations of bulk thermoelectrics. Here, we demonstrate that the $\mathrm{Bi}_{0.5} \mathrm{Sb}_{1.5} \mathrm{Te}_{3}$ (BST) epitaxial thin film on sapphire substrate grown by spontaneous van der Waals epitaxy (vdWE) is exfoliated and transferred onto versatile materials, creating the high-quality freestanding thermoelectric membranes. Unprecedented millimeter-size vdWE BST membranes are produced by etching pseudomorphic Te monolayer on the surface of sapphire substrate in dilute HF solution. The intact exfoliation and direct transfer for vdWE BST membranes ensures a high thermoelectric performance, maintaining the high-quality crystallinity and subsequently showing the remarkable $z T$ value $(\sim 0.9$ at $300 \mathrm{~K})$ in thin film thermoelectrics. These results represent the realization of long pursued but yet to be demonstrated high-performance thin film thermoelectrics, paving the way for design and fabrication of arbitrary shaped thermoelectric devices.

\section{Introduction}

With the increasing demand of sustainable and environmentally friendly energy supply, thermoelectrics have attracted a great interest in diverse fields ranging from electronics to energy storage and conversion because they can directly convert thermal energy to electricity ${ }^{1}$. Compared with bulk thermoelectrics, which have been widespread by using a flat and rigid module device ${ }^{2-4}$, thin film thermoelectrics have shown many attractive advantages such as micro-cooling ability for solving heat dissipation issues in electronics and energy scavenging from human body heat ${ }^{5-7}$. Indeed, thin film thermoelectrics have a potential for curved and flexible module devices, which promise a versatile energy harvesting from the close contact to various arbitrary geometries of heat sources ${ }^{6,8-11}$. While organic thin film thermoelectrics have a relevance for such devices, a low thermoelectric performance has been a major obstacle ${ }^{12,13}$. This provokes the inevitable use of inorganic $\mathrm{Bi}_{2} \mathrm{Te}_{3}$-based state-of-the-art materials for the curved and flexible thermoelectrics ${ }^{8,14-16}$, but remains challenging due to the anisotropic brittle crystal and mechanical incompatibility.

Thermoelectric $\mathrm{Bi}_{2} \mathrm{Te}_{3}$-based thin films have been achieved by the conventional heterogeneous epitaxial growth on single crystalline substrates with a small lattice mismatch and atomically flat surface ${ }^{17,18}$, which suppress the formation of structural and atomic defects. However, this heterogenous epitaxial thin film growth limits the applications to the curved and flexible thermoelectrics due to the clamping effect, inherited from strong bond between overlayer film and substrate at interface, of epitaxial thin films on the flat substrate ${ }^{19,20}$. Furthermore, it is extremely difficult to grow high quality epitaxial thin films on the materials with large lattice mismatch or on the flexible matters ${ }^{21}$. These obstacles have been solved by 
using the flexible scaffold materials such as carbon nanotube anchored with highly oriented $\mathrm{Bi}_{2} \mathrm{Te}_{3}$ nanocrystals, providing a promising way for practical flexible thermoelectric devices ${ }^{22}$. Out of heterogeneous epitaxy scheme, the van der Waals epitaxy (vdWE) has proved an efficient growth of highperformance $\mathrm{Bi}_{2} \mathrm{Te}_{3}$-based thin films on the various substrates ${ }^{23-25}$. Indeed, the layer structured $\mathrm{Bi}_{0.5} \mathrm{Sb}_{1.5} \mathrm{Te}_{3}$ (BST) alloy, which are widespread p-type material for room temperature refrigeration and power generation, has been successfully grown via the vdWE on the graphene transferred amorphous silica substrate and on the pseudomorphic Te monolayer at the surface of sapphire substrate, showing comparable electrical properties to single crystal BST alloy. Considering the previous lift-off techniques for freestanding complex-oxide membranes ${ }^{20}$, this vdWE BST thin film, which has a weak bonding at the vdW hetero-interface between Te-terminated quintuple layer and pseudomorphic Te monolayer on sapphire substrate, has a potential for intact exfoliation and direct transfer on curved or flexible supports for freestanding thermoelectric membranes. The naturally-formed pseudomorphic Te monolayer on the sapphire substrate during in-situ spontaneous vdWE growth is of benefit in practical lift-off process in chemical solution, working as a sacrificial layer. Additionally, the sacrificial Te monolayer is expected to

have a high etch selectivity, allowing to overcome the well-known shortcoming, a slow release rate ${ }^{21,26}$, of chemical lift-off for larger substrates.

\section{Results}

Here, we demonstrate a high-performance freestanding BST thermoelectric membrane on curved glass and flexible polyethylene terephthalate (PETE) (Supplementary Fig. 1), in which the vdWE BST thin films grown by pulsed laser deposition (PLD) method are intactly separated from sapphire substrate and transferred onto various supporters using chemical lift-off technique (Fig. 1). First, we prepared vdWE BST thin film on the sapphire substrate by spontaneous vdW epitaxy ${ }^{25}$. As highlighted, the in-situ formation of pseudomorphic Te monolayer on the surface of sapphire substrate is the key in the growth of VdWE BST thin films, allowing the weak vdW bonding between the Te monolayer and the outermost Te atomic layer of the Te-Bi/Sb-Te-Bi/Sb-Te quintuple layer (Fig. 1a). Importantly, this diminishes a strong clamping effect between film and substrate in the growth of heterogeneous epitaxial films and promises an easy exfoliation by chemical etching of the Te monolayer or mechanical peeling-off technique using scotch tape. While the latter mechanical method requires a special process such as thermal heating and additional metal layer deposition, the former can allow the selective etching of Te monolayer in a dilute $\mathrm{HF}$ solvent. For preserving the epitaxial BST thin film during the chemical etching of the sacrificial Te monolayer, approximately $100 \mathrm{~nm}$ thick polymethyl methacrylate (PMMA) was spin-coated on the top of the BST thin film (Fig. 1b, c). When the etching is completed, the thin PMMA-capped BST film is exfoliated from sapphire (Supplementary Fig. 2) and becomes floating in the solvent. Once the BST film with PMMA overlayer is transferred onto other substrates, the PMMA overlayer is completely removed using acetone, IPA, and DI water. To verify the suggested concept and methodology for transferring vdWE BST films, $\mathrm{SiO}_{2} / \mathrm{Si}$ wafer, flat and curved glasses, and flexible polyethylene terephthalate (PETE) films were chosen for the destination substrates (Fig. 1e). A strong adhesion to the substrates was achieved by 
baking at 373-393 K, ending the whole procedures for creating the first freestanding thermoelectric membranes.

Benefiting from the sacrificial pseudomorphic Te monolayer, the crystallinity of epitaxial BST thin film can be maintained during the transfer as shown by the scanning electron microscopy (SEM) observations and X-ray diffraction (XRD) measurements (Fig. 2). SEM cross-sectional images clearly show that the nano-grained structure of epitaxial BST thin film is hardly damaged by the chemical etching (Fig. $2 d-f$ ). We conducted XRD $f$ and $w$ scans for the as-grown vdWE BST thin film on sapphire substrate and the transferred BST membranes on flat glass and flexible PETE (Fig. $2 \mathrm{~g}-\mathrm{i}$ and Supplementary Fig. 3). The $f$ scans of (105) reflections for the all samples (Fig. 2g-i) exhibit a six-fold symmetry, indicating that the inplane crystallinity of epitaxial BST thin film is well maintained in the transferred membranes on both flat glass and flexible PETE supports without any collapse of expitaxy. Except the diffraction peaks of substrates, all samples show only intense (00/) peaks of BST structure (Supplementary Fig. 2). Additionally, the $w$ rocking curves of the (0015) peak (Supplementary Fig. 4), which show the decreased peak intensity and broadened FWHM of the transferred membranes on the curved glass and flexible PETE, implying that the out-of-plane crystallinity of the transferred membranes slightly degrades due to the inherent brittleness of inorganic BST alloy.

The crystallinity and composition of the transferred BST membranes are also examined by the highresolution scanning transmission electron microscopy (HR-STEM). Figure 3a shows low magnification top-view high-angle annular dark field (HAADF) image of exfoliated BST membrane. The nano-domain structure is consistent with those observed in cross-sectional SEM images. Low magnification energy dispersive spectroscopy (EDS) analysis shows the $\mathrm{Bi}, \mathrm{Sb}$ and Te elements are homogeneously distributed in the membrane, indicating that no impurity phase occurs during the chemical etching and transfer (Fig. $3 \mathrm{~b}-\mathrm{e}$ ). Furthermore, cross-sectional image of transferred BST membrane on $\mathrm{SiO}_{2} / \mathrm{Si}$ wafer clearly evidences the intact transfer of epitaxial BST thin film, maintaining the layered structure with quintuple BST layers in the membrane (Fig. 3f, g). A high crystallinity of BST membrane is confirmed by the perfect $\mathrm{Te}(1)-\mathrm{Bi} / \mathrm{Sb}-\mathrm{Te}(2)-\mathrm{Bi} / \mathrm{Sb}-\mathrm{Te}$ (1) quintuple layers separated by vdW gaps along [00/ direction (Fig. $3 \mathrm{~g}$ ) and by the selected area electron diffraction (SAED) patterns along [001] and [120] zone axes (insets of Fig. $3 a, f)$. Most of all, note that the pseudomorphic Te monolayer formed in the growth of vdWE BST thin film is completely removed by the chemical etching, showing no trace of Te monolayer on the $\mathrm{SiO}_{2} / \mathrm{Si}$ wafer (Fig. 3g, Supplementary Figs. 5 and 6 and Supplementary Note 1). Atomic-scale STEM-EDS mappings for constituent $\mathrm{Bi}, \mathrm{Sb}$ and Te atoms also demonstrate that the quintuple layers are well-separated by vdW gaps along $c$-axis and typical atomic-scale defects such as $\mathrm{Bi}(\mathrm{Sb}) / \mathrm{Te}$ antisite defect are hardly found. These structural features, as represented by the nano-grained structure with a high crystallinity (Fig. 3a, b), give an expectation for a high power factor $(P F)$ comparable to that of single crystal and a low 
thermal conductivity similar to the nanostructured BST bulk, enabling a high performance thermoelectric freestanding BST membrane.

Thermoelectric transport properties are measured along the in-plane direction for as-grown epitaxial BST films and transferred BST membranes at room temperature (Fig. 4). The room-temperature electrical conductivity $(s)$ and Seebeck coefficient $(S)$ values of as-grown epitaxial BST films are ranged in 1300$1700 \mathrm{~s} \mathrm{~cm}^{-1}$ and $140-170 \mathrm{mV} \mathrm{K}^{-1}$, respectively, resulting the $P F$ of $3.0-4.0 \mathrm{~mW} \mathrm{~m}^{-1} \mathrm{~K}^{-2}$ at $300 \mathrm{~K}$. The $s$ values of the transferred membranes increased to $\sim 1900 \mathrm{~S} \mathrm{~cm}^{-1}$ on flat glass and decreased to $1100 \mathrm{~S}$ $\mathrm{cm}^{-1}$ on curved glass and $\sim 1250 \mathrm{~S} \mathrm{~cm}^{-1}$ on flexible PETE (Fig. 4a). Accordingly, average roomtemperature $S$ values showed the trade-off relation (Fig. 4a). The average $P F$ values were ranged in 2.5$3.4 \mathrm{~mW} \mathrm{~m}^{-1} \mathrm{~K}^{-2}$ for the transferred membranes (Fig. $4 \mathrm{~b}$ ). We have measured the out-of-plane thermal conductivity $\left(k_{\text {out }}\right)$ by using a conventional $2 w$ method as shown in the inset of Fig. $4 \mathrm{c}$. To estimate the in-plane thermal conductivity $\left(k_{\text {in }}\right)$, we used the reported anisotropy ratio of $k_{\text {in }} / k_{\text {out }}$ in bulk single crystalline BST is $\sim 2.0^{27}$ for the as-grown films and transferred membranes owing to the high quality crystallinity. The $k_{\text {out }}$ and $k_{\text {in }}$ values of transferred membranes show the similar values with as-grown vdWE films. The estimated $k_{\text {in }}$ values are lower than that of bulk single crystalline BST due to the nanogained structure. In-plane lattice thermal conductivity $\left(k_{\mathrm{L}, \mathrm{in}}\right)$ for as-grown films and transferred membranes on flat and curved glass substrates were calculated by subtracting the electronic part from $k_{\text {in }}$ (see Supplementary Note 2 and Supplementary Fig. 7 for details). The $k_{\text {L,in }}$ values for all the films and membranes are $\sim 0.4 \mathrm{~W} \mathrm{~m}^{-1} \mathrm{~K}^{-1}$, which is slightly higher than that $\left(\sim 0.3 \mathrm{~W} \mathrm{~m}^{-1} \mathrm{~K}^{-1}\right)$ of nanostructured bulk BST with the high-density dislocation array at grain-boundary ${ }^{28}$. This indicates that the in-plane thermal conduction suffers from the intensive phonon scattering at boundaries between nano-sized mosaic domains as shown in Fig. 3a, b. The resultant thermoelectric figure of merit $z T\left(=s S^{2} T / k\right.$, where $T$ is absolute temperature and $k$ is the total thermal conductivity) values are shown in Fig. $4 \mathrm{~d}$ with the reported $z T$ values of $\mathrm{Bi}_{2} \mathrm{Te}_{3}$-based thin films for comparison. The in-plane $z T$ values for as-grown epitaxial BST films are ranged in 1.0 1.6, which is comparable to that of state-of-the-art nanostructure bulk $\mathrm{BST}^{29}$ and is the highest value in the reported BST thin film ${ }^{6,22,30,31}$. Moreover, the estimated roomtemperature $z T$ values for the transferred BST membranes are comparable to those of zone melted BST ingots used in the commercialized modules ${ }^{32,33}$. These values are also comparable to those of highperformance BST thin films on the rigid substrates, proving the merit of present lift-off and transferring processes for the vdWE thin films. By considering the projected efficiency of $4.6 \%$ of the transferred membranes (see Supplementary Note 3 for details) when the cold- and hot-side temperature are $300 \mathrm{~K}$ and $350 \mathrm{~K}$, respectively, the present work is promising for the diverse arbitrary thermoelectric devices.

Among the transferred membranes, we conducted the bending test of the membrane on flexible PETE (Fig. 5). From the original $s, S$ and $P F$ values of vdWE thin film as a reference, all transport parameters of 
the membrane on flexible PETE, its bended state with a radius of $15 \mathrm{~mm}$ and flattened state showed no obvious changes but slight decreases (Fig. $5 b-d$ ), which are mainly attributed to the decrease of $s$ coming from the generation of structural microcracks (Supplementary Fig. 8). This unfavorable structural defect in the membrane is accelerated in the cyclic flexible bending tests. As shown in Fig. $5 \mathrm{e}-\mathrm{g}$, the $S$ values upon bending cycles are almost unchanged while $s$ values are largely degraded, leading to the loss of $40 \%$ of original PFat 100 bending cycles in a bending radius of $\sim 10 \mathrm{~mm}$. As observed microcracks in the bended membranes, a bending deformation creating a high density microcrack is responsible for the decreased performance in the membranes on flexible PETE. However, it is apparent that there is sustainable performance, which was found from the membranes on curved glass, strongly suggesting that the present millimeter-sized membranes are more appropriate for the application of curved surface of heat source rather than the flexible thermoelectric devices.

\section{Discussion}

In summary, we developed a strategy to fabricate high-performance freestanding thermoelectric membranes from the chemical exfoliation of high-quality vdWE BST thin films on sapphire and intact transfer onto versatile substrates. Although the millimeter-sized BST membranes are difficult to apply for the flexible thermoelectric devices, our demonstration is promising for the practical devices on the curved surface of versatile heat source such as human body. The $z T$ value of $\sim 0.9$ at $300 \mathrm{~K}$, which is comparable or much higher than those of previous BST-based films, will stimulate the fabrication of highperformance n-type $\mathrm{Bi}_{2} \mathrm{Se}_{2.7} \mathrm{Te}_{0.3}$ thermoelectric membranes using this method. Our demonstrations advance thermoelectric thin film research by allowing unrestricted transfer onto diverse substrates for freestanding membranes.

\section{Methods}

Material preparation. $\mathrm{Bi}_{0.5} \mathrm{Sb}_{1.5} \mathrm{Te}_{3}$ films were deposited on a- $\mathrm{Al}_{2} \mathrm{O}_{3}(001)$ single crystal substrate (10 ' 10 $\mathrm{mm}^{2}$ ) by PLD with the fluence of $1.2-1.50 \mathrm{~J} \mathrm{~cm}^{-2}$ of $\mathrm{KrF}$ excimer laser ( $/=248 \mathrm{~nm}$ ) using a 2-inch diameter $\mathrm{Bi}_{0.5} \mathrm{Sb}_{1.5} \mathrm{Te}_{3}$ target, while the target were prepared in the laboratory by using conventional solid state reaction followed by consolidation by spark plasma sintering. The substrate temperature was set at $200-230^{\circ} \mathrm{C}$, while the argon partial pressure was at 10 mTorr. Films were deposited with $\sim 130 \mathrm{~nm}$ in thicknesses. XRD analyses of $2 q, w$ and $f$ scans for the films using X-ray diffractometer (Rigaku Smartlab, Rigaku Co.) with Cu Ka1 radiation ( $=1.540592 \AA)$.

Thermoelectric properties measurement. The in-plane $s$ and $S$ were measured at room temperature by ZEM-3 system (Advanced Riko, Japan). The out-of-plane $k_{\text {out }}$ of the films were measured with the $2 w$ method (TCN-2 $w$, Advanced Riko, Japan). The Hall measurement was performed by using the van der Pauw method (8400 HMS, Lake Shore Cryotronics, Inc., USA). 
Microstructure characterization. The top and cross section morphology of as grown BST film and BST film transferred to flat glass substrate were analyzed by SEM equipped with an EDS detector (FESEM, JSM-7000F, JEOL, Japan). Exfoliated film for STEM was prepared using either a dual-beam focused ion beam (AURIGA CrossBeam Workstation, Carl Zeiss, Germany) or direct pulverizing by super sonication. The HAADF-STEM images were obtained from a probe-corrected STEM (JEM-ARM200F, JEOL, Japan) equipped with a cold field emission source, operating at $200 \mathrm{kV}$. To improve the image resolution, successive images were acquired with short time intervals and averaged for the same area.

\section{Data availability}

The data that supports the findings of this study are available from the corresponding author upon reasonable request.

\section{References}

1. Zhu, T., Liu, Y., Fu, C., Heremans, J. P., Snyder, J. G. \& Zhao, X. Compromise and synergy in highefficiency thermoelectric materials. Adv. Mater. 29, 1605884 (2017).

2. $\mathrm{Hu}, \mathrm{X}$., et al. Power generation from nanostructured PbTe-based thermoelectrics: comprehensive development from materials to modules. Energy Environ. Sci. 9, 517-529 (2016).

3. Kraemer, D., et al. Concentrating solar thermoelectric generators with a peak efficiency of $7.4 \%$. Nat. Energy 1, 1-8 (2016).

4. Zhang, Q., et al. Realizing a thermoelectric conversion efficiency of $12 \%$ in bismuth telluride/skutterudite segmented modules through full-parameter optimization and energy-loss minimized integration. Energy Environ. Sci. 10, 956-963 (2017).

5. Chowdhury, I., et al. On-chip cooling by superlattice-based thin-film thermoelectrics. Nat. Nanotechnol. 4, 235-238 (2009).

6. Kim, S. J., We, J. H. \& Cho, B. J. A wearable thermoelectric generator fabricated on a glass fabric. Energy Environ. Sci. 7, 1959-1965 (2014).

7. Jiang, C., et al. Ultrahigh performance of n-Type $\mathrm{Ag}_{2} \mathrm{Se}$ films for flexible thermoelectric power generators. ACS Appl. Mater. Interfaces 12, 9646-9655 (2020).

8. Hou, W., et al. Fabrication and excellent performances of $\mathrm{Bi}_{0.5} \mathrm{Sb}_{1.5} \mathrm{Te}_{3}$ /epoxy flexible thermoelectric cooling devices. Nano Energy 50, 766-776 (2018).

9. Vieira, E. M. F., et al. High-performance $\mu$-thermoelectric device based on $\mathrm{Bi}_{2} \mathrm{Te}_{3} / \mathrm{Sb}_{2} \mathrm{Te}_{3} \mathrm{p}-\mathrm{n}$ junctions. ACS Appl. Mater. Interfaces 11, 38946-38954 (2019).

10. Lu, Y., et al. Ultrahigh power factor and flexible silver selenide-based composite film for thermoelectric devices. Energy Environ. Sci. 13, 1240-1249 (2020). 
11. Shang, $\mathrm{H}$., et al. $\mathrm{Bi}_{0.5} \mathrm{Sb}_{1.5} \mathrm{Te}_{3}$-based films for flexible thermoelectric devices. J. Mater. Chem. A 8 , 4552-4561 (2020).

12. Norton-Baker, B., et al. Polymer-free carbon nanotube thermoelectrics with improved charge carrier transport and power factor. ACS Energy Lett. 1, 1212-1220 (2016).

13. Li, D., et al. High performance polymer thermoelectric composite achieved by carbon-coated carbon nanotubes network. ACS Appl. Energy. Mater. 2, 2427-2434 (2019).

14. Jeong, S. H., et al. Stretchable thermoelectric generators metallized with liquid alloy. ACS Appl. Mater. Interfaces, 9, 15791-15797 (2017).

15. Kim, C. S., et al. Self-powered wearable electrocardiography using a wearable thermoelectric power generator. ACS Energy Lett. 3, 501-507 (2018).

16. Hong, S., et al. Wearable thermoelectrics for personalized thermoregulation. Sci. Adv. 5, eaaw0536 (2019).

17. Peranio, N., Eibl O. \& Nurnus, J. Structural and thermoelectric properties of epitaxially grown $\mathrm{Bi}_{2} \mathrm{Te}_{3}$ thin films and superlattices. J. Appl. Phys. 100, 114306 (2006).

18. Fornari, C. I., Rappl, P. H. O., Morelhão, S. L. \& Abramof, E. Structural properties of $\mathrm{Bi}_{2} \mathrm{Te}_{3}$ topological insulator thin films grown by molecular beam epitaxy on (111) $\mathrm{BaF}_{2}$ substrates. J. Appl. Phys. 119, 165303 (2016).

19. Sun, $\mathrm{T}$., et al. Enhanced thermoelectric properties of $\mathrm{n}$-type $\mathrm{Bi}_{2} \mathrm{Te}_{2.7} \mathrm{Se}_{0.3}$ thin films through the introduction of Pt nanoinclusions by pulsed laser deposition. Nano Energy 8, 223-230 (2014).

20. Kum, H. S., et al. Heterogeneous integration of single-crystalline complex-oxide membranes. Nature 578, 75-81 (2020).

21. Kum, H., et al. Epitaxial growth and layer-transfer techniques for heterogeneous integration of materials for electronic and photonic devices. Nat. Electron. 2, 439-450 (2019).

22. Jin, Q., et al. Flexible layer-structured $\mathrm{Bi}_{2} \mathrm{Te}_{3}$ thermoelectric on a carbon nanotube scaffold. Nat. Mater. 18, 62-68 (2019).

23. Kampmeier, J., Borisova, S., Plucinski, L., Luysberg, M., Mussler, G. \& Grützmacher, D. Suppressing twin domains in molecular beam epitaxy grown $\mathrm{Bi}_{2} \mathrm{Te}_{3}$ topological insulator thin films. Cryst. Growth Des. 15, 390-394 (2015).

24. Kim, E. S., Hwang, J.-Y., Lee, K. H., Ohta, H., Lee, Y. H. \& Kim, S. W. Graphene substrate for van der Waals epitaxy of layer-structured bismuth antimony telluride thermoelectric film. Adv. Mater. 29, 1604899 (2016).

25. Hwang, J.-Y., Kim, Y.-M., Lee, K. H., Ohta, H. \& Kim, S. W. Te Monolayer-driven spontaneous van der Waals epitaxy of two-dimensional pnictogen chalcogenide film on Sapphire. Nano Lett. 17, 61406145 (2017).

26. Cheng, C.-W., Shiu, K.-T., Li, N., Han, S.-J., Shi, L. \& Sadana, D. K. Epitaxial lift-off process for gallium arsenide substrate reuse and flexible electronics. Nat. Commun. 4, 1577 (2013). 
27. Shen, J. J., Hu, L. P., Zhu, T. J. \& Zhao, X. B. The texture related anisotropy of thermoelectric properties in bismuth telluride based polycrystalline alloys. Appl. Phys. Lett. 99, 124102 (2011).

28. Kim, S. I., et al. Dense dislocation arrays embedded in grain boundaries for high-performance bulk thermoelectrics. Science 348, 109-114 (2015).

29. Poudel, B., et al. High-thermoelectric performance of nanostructured bismuth antimony telluride bulk alloys. Science 320, 634-638 (2008).

30. Takashiri, M., Tanaka, S. \& Miyazaki, K. Improved thermoelectric performance of highly-oriented nanocrystalline bismuth antimony telluride thin films. Thin Solid Films 519, 619-624 (2010).

31. Du, Y., Cai, K. F., Chen, S., Cizek, P. \& Lin, T. Facile preparation and thermoelectric properties of $\mathrm{Bi}_{2} \mathrm{Te}_{3}$ based alloy nanosheet/PEDOT:PSS composite films. ACS Appl. Mater. Interfaces 6, 5735-5743 (2014).

32. Zheng, Y., Xie, H., Shu, S., Yan, Y., Li, H. \& Tang, X. High-temperature mechanical and thermoelectric properties of p-yype $\mathrm{Bi}_{0.5} \mathrm{Sb}_{1.5} \mathrm{Te}_{3}$ commercial zone melting ingots. J. Electron. Mater. 43, 2017-2022 (2014).

33. Zheng, Y., et al. Mechanically robust BiSbTe alloys with superior thermoelectric performance: A case study of stable hierarchical nanostructured thermoelectric materials. Adv. Energy Mater. 5, 1401391 (2015).

\section{Declarations}

\section{Acknowledgements}

This work was supported by the Institute for Basic Science (IBS-R011-D1), Creative Materials Discovery Program through the National Research Foundation of Korea (NRF) grant (NRF-2015M3D1A1070672), and the NFR grant (No. 2017R1A2B3011949) funded by the Korean government in Korea.

\section{Author contributions}

S.W.K conceived the idea and designed experiments. L.F, K.P., K.H.L., B.K, S.I.K and J.-Y.H, carried out the experimental work, including sample preparation and thermoelectric performance measurements. W.C and Y.M.K carried out microstructure measurements. All authors discussed the results and commented on the paper. All of authors co-wrote the paper.

\section{Competing interests}

The authors declare no conflict of interest. 
Correspondence and requests for materials should be addressed to S.I.K., J.-Y.H. and S.W.K.

\section{Figures}

a vdW epitaxial BST film grown by pulsed laser deposition (PLD)

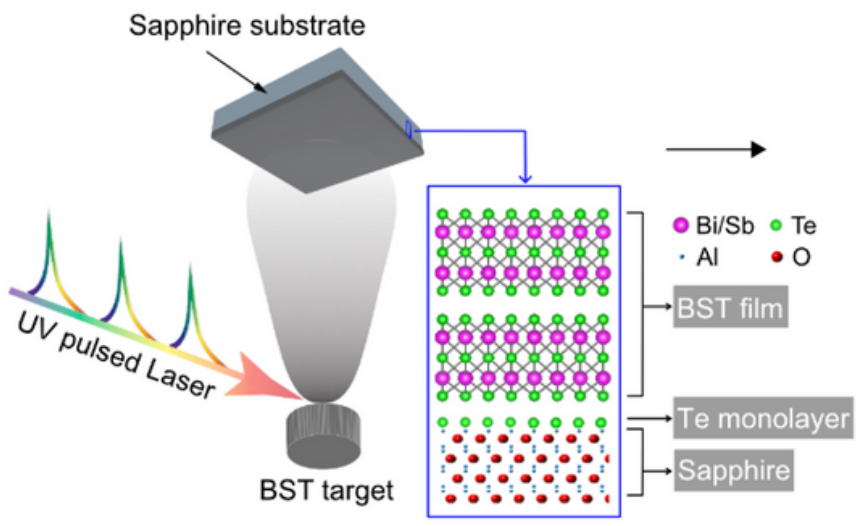

b PMMA spin coating

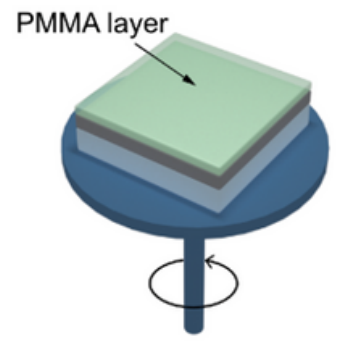

f BST membranes on various substrates

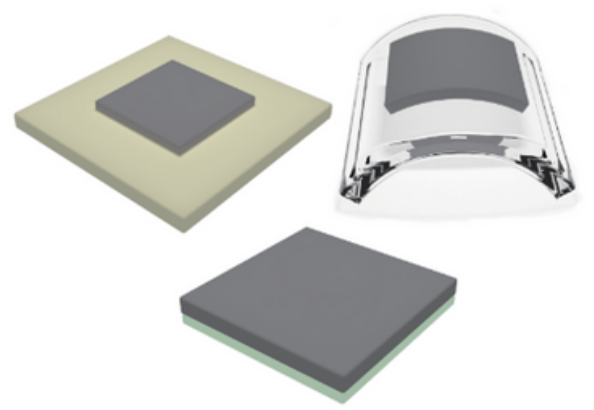

e Transferred BST membrane with PMMA on flat or curved substrates
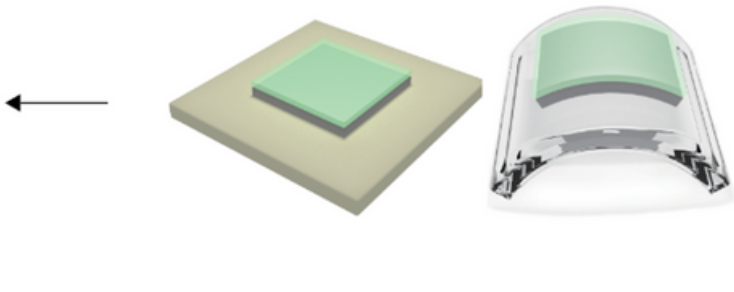

d Freestanding BST membrane with PMMA support

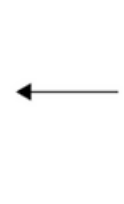

c Peeling-off in dilute HF solution

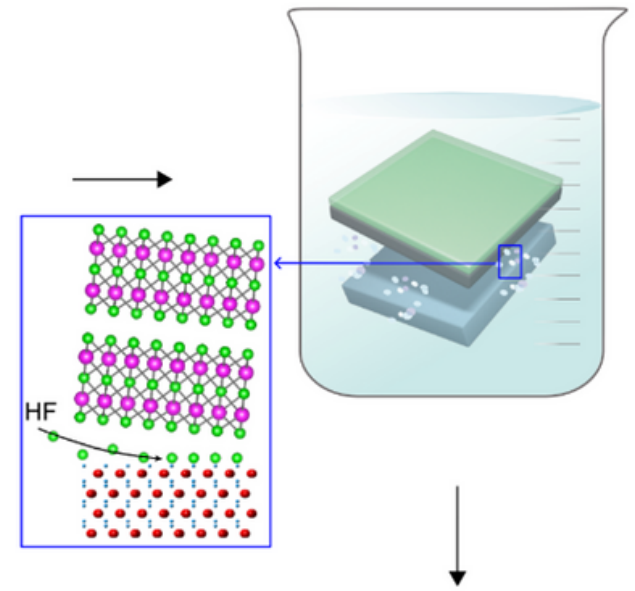

\section{Figure 1}

Exfoliation and transfer of vdW epitaxial (vdWE) Bi0.5Sb1.5Te3 (BST) thin film. a vdWE BST thin film on sapphire substrate grown by pulsed laser deposition (PLD). The schematic plot shows clearly the Te monolayer between the BST film and sapphire substrate. b Spin coating of PMMA layer on as-grown vdWE BST film. c Peeling-off of vdWE BST film from sapphire substrate in the dilute HF solution. The schematic illustration shows that the Te monolayer is selectively etched by HF. $d$ Freestanding BST membrane with PMMA overlayer. e Transferring PMMA supported BST membrane on flat or curved substrate. $\mathrm{f} \mathrm{BST}$ membrane on various substrates with/without removing the PMMA layer. 
a vdW epitaxial BST film grown by pulsed laser deposition (PLD)

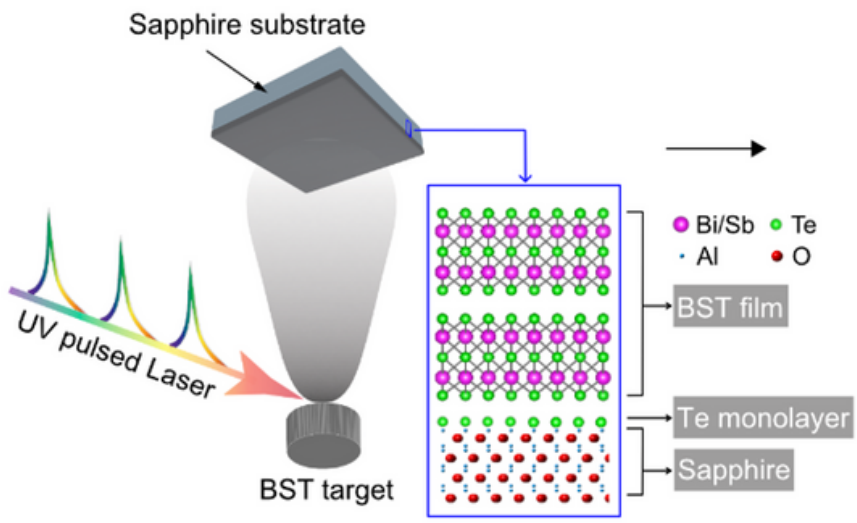

b PMMA spin coating

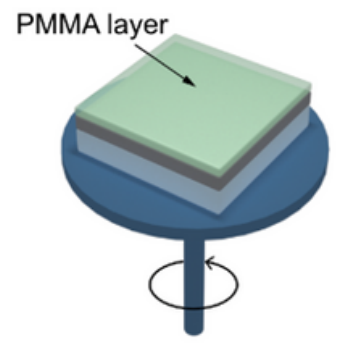

f BST membranes on various substrates

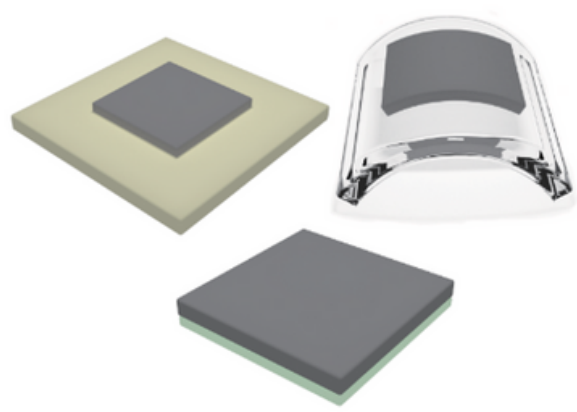

e Transferred BST membrane with PMMA on flat or curved substrates
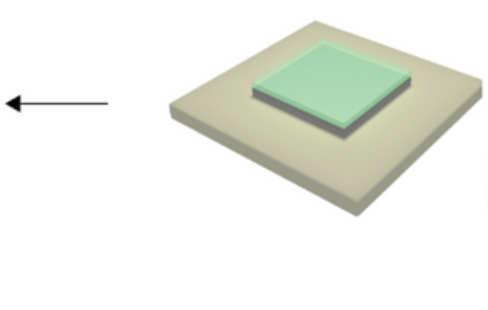

d Freestanding BST membrane with PMMA support

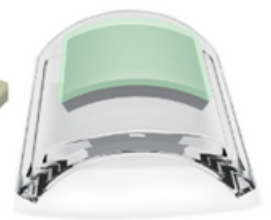

C Peeling-off in dilute $\mathrm{HF}$ solution

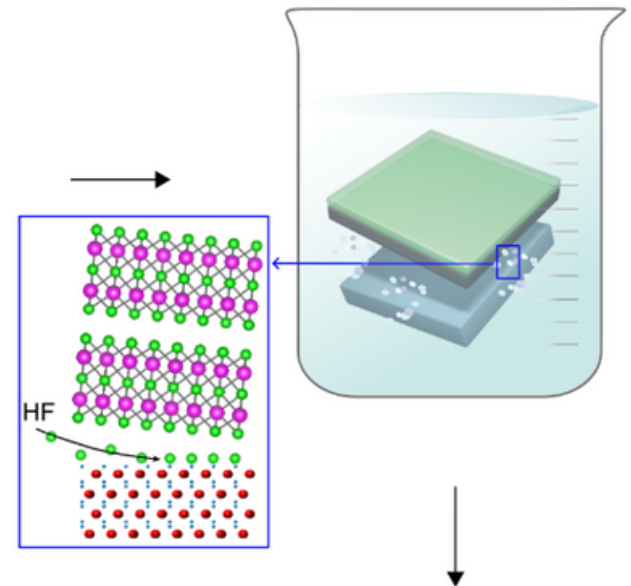

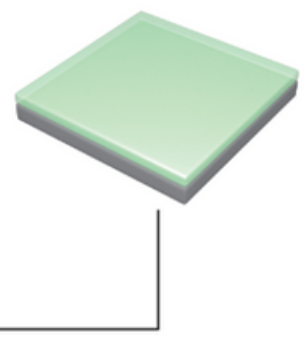

\section{Figure 1}

Exfoliation and transfer of vdW epitaxial (vdWE) Bi0.5Sb1.5Te3 (BST) thin film. a vdWE BST thin film on sapphire substrate grown by pulsed laser deposition (PLD). The schematic plot shows clearly the Te monolayer between the BST film and sapphire substrate. b Spin coating of PMMA layer on as-grown vdWE BST film. c Peeling-off of vdWE BST film from sapphire substrate in the dilute HF solution. The schematic illustration shows that the Te monolayer is selectively etched by HF. $d$ Freestanding BST membrane with PMMA overlayer. e Transferring PMMA supported BST membrane on flat or curved substrate. $f$ BST membrane on various substrates with/without removing the PMMA layer. 
a

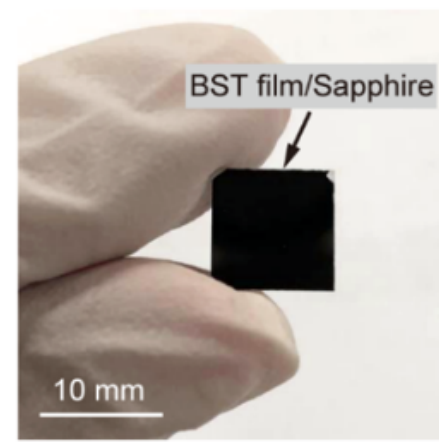

b

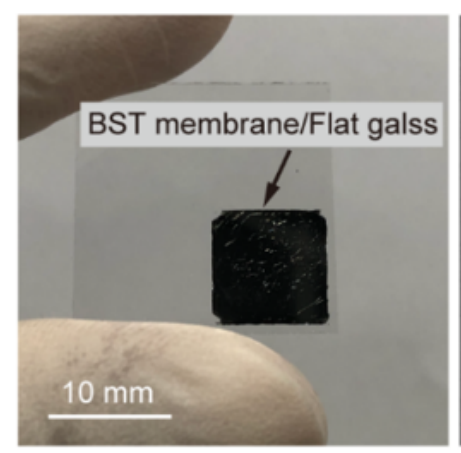

c

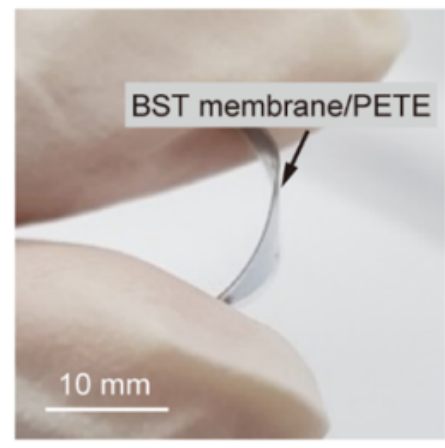

d

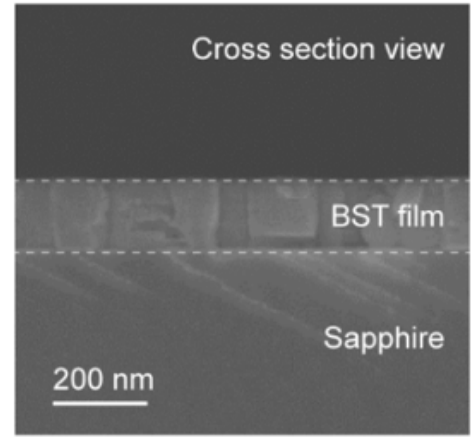

e

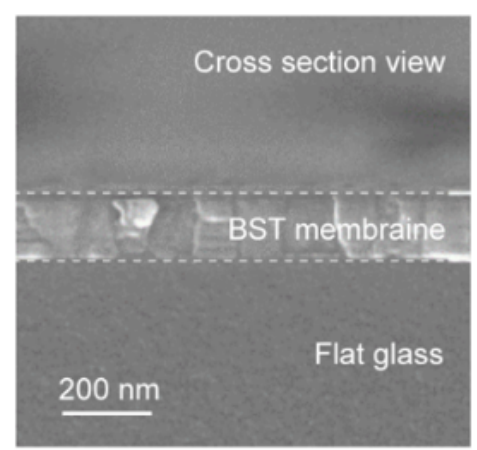

$200 \mathrm{~nm}$

Top view

$\underline{200 \mathrm{~nm}}$

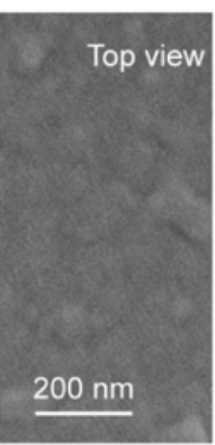

f

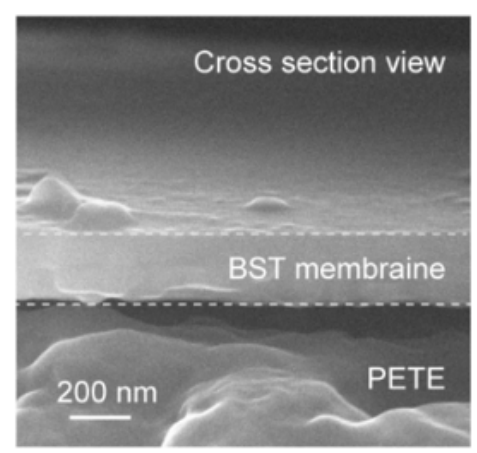

g

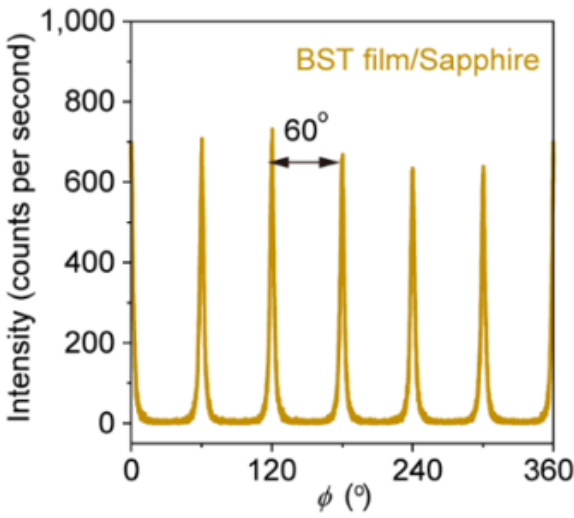

h
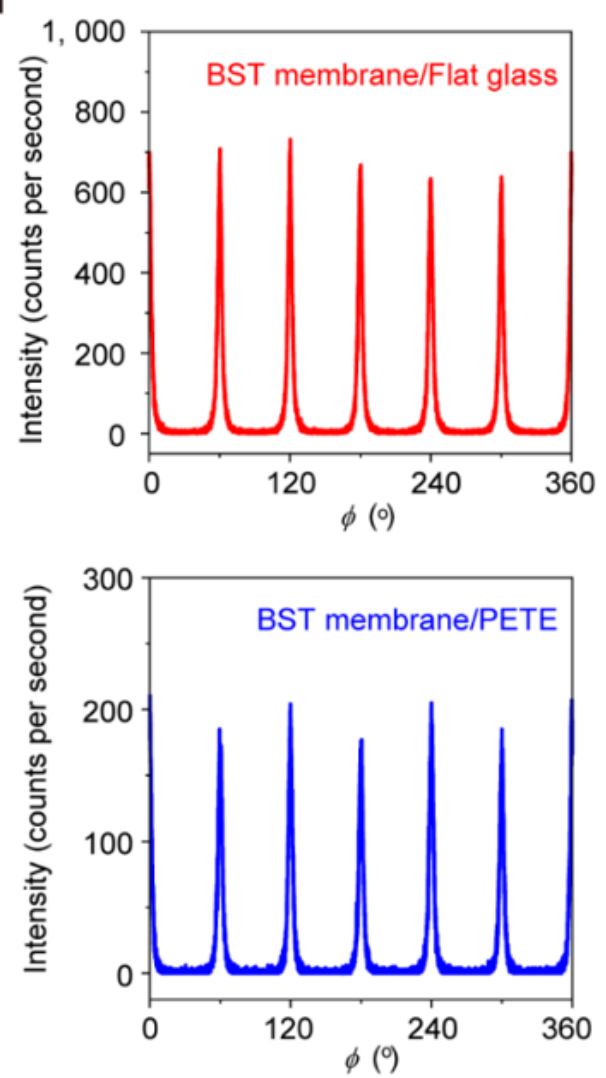

Figure 2

Morphology and XRD analysis of as-grown vdWE BST film and transferred BST membranes on flat glass and flexible PETE. a-c Photos and top-view SEM images of as-grown BST film (a) and transferred BST membranes on flat glass (b) and PETE (c) substrates. $d-f$ Cross-section SEM images of the vdWE BST films (d) and transferred BST membranes on glass (e) and PETE ( $f$ ) substates. $g-i \otimes$ scan measured by XRD for as-grown vdWE BST film (g) and transferred BST membranes on flat glass $(\mathrm{h})$ and flexible PETE (i), respectively. 
a

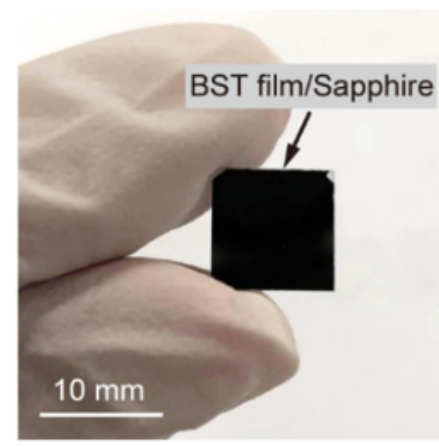

b

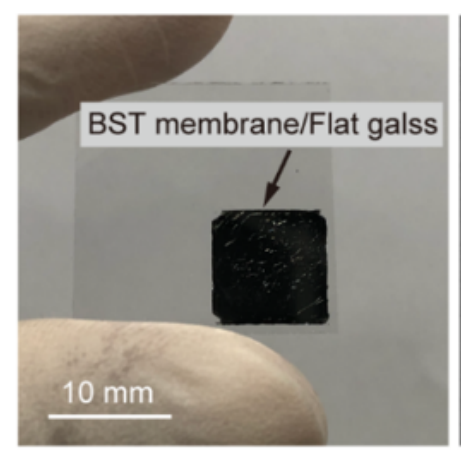

c

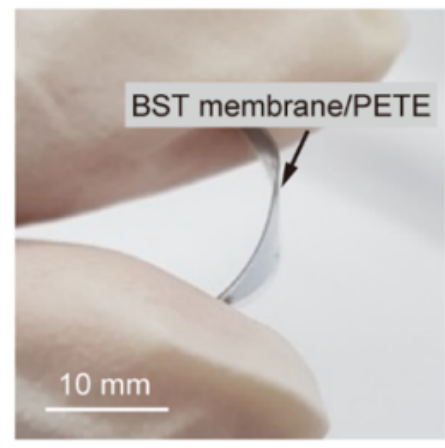

d

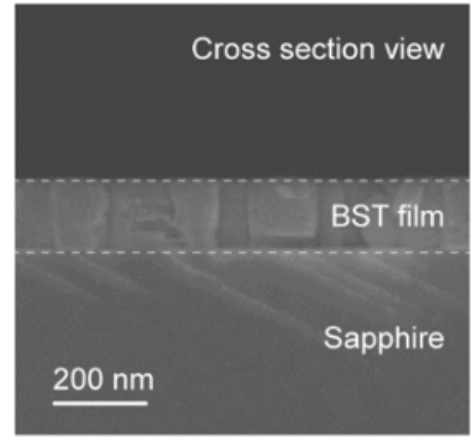

e

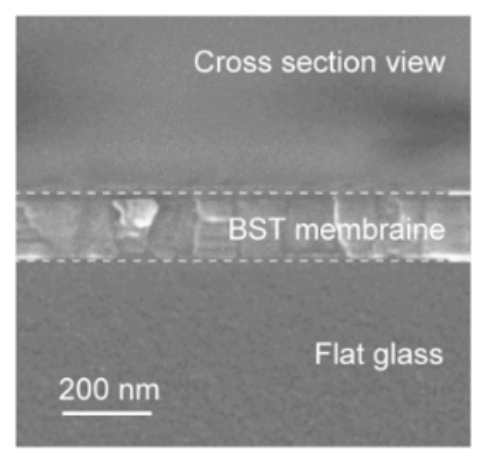

$200 \mathrm{~nm}$

Top view

$\underline{200 \mathrm{~nm}}$

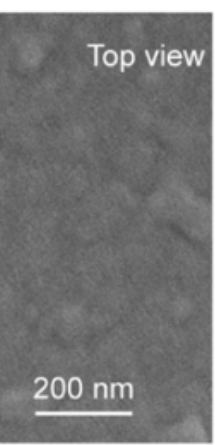

f

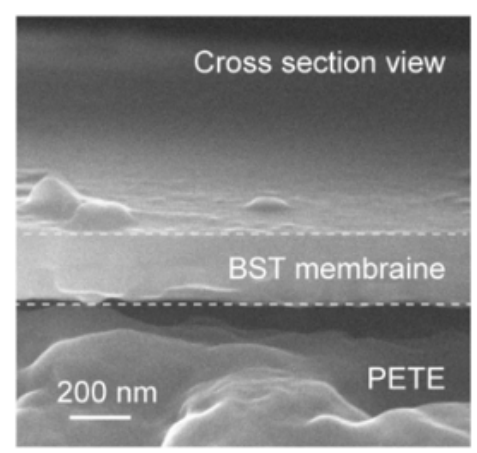

g

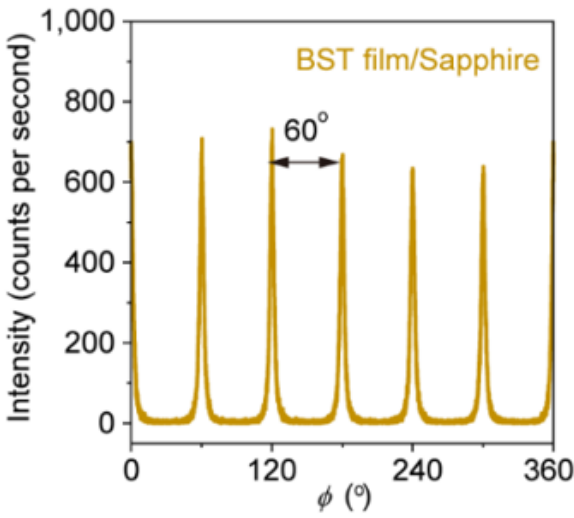

h
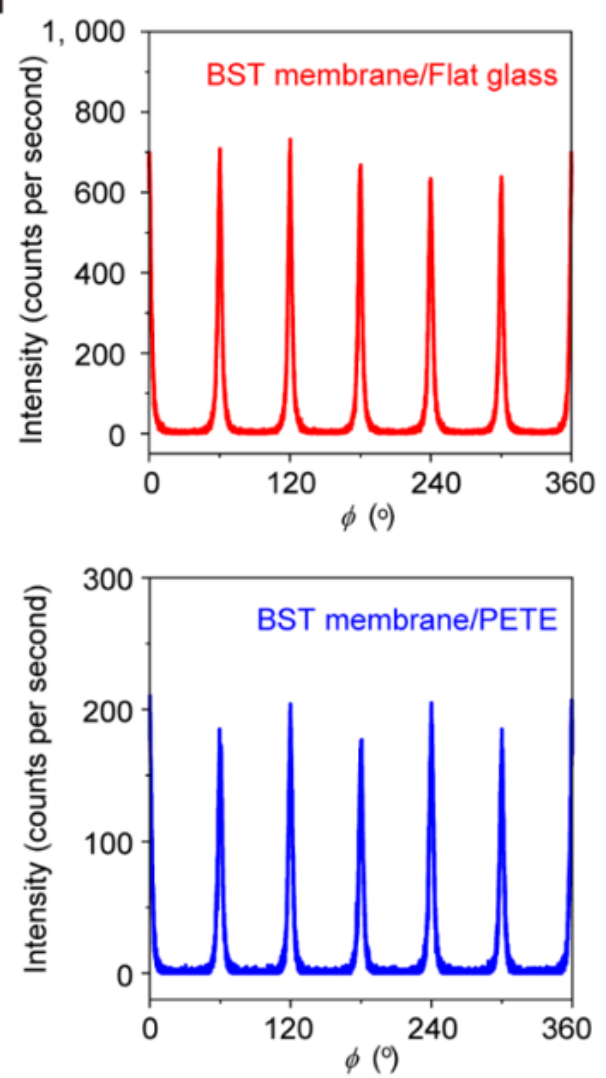

Figure 2

Morphology and XRD analysis of as-grown vdWE BST film and transferred BST membranes on flat glass and flexible PETE. a-c Photos and top-view SEM images of as-grown BST film (a) and transferred BST membranes on flat glass (b) and PETE (c) substrates. $d-f$ Cross-section SEM images of the vdWE BST films (d) and transferred BST membranes on glass (e) and PETE ( $f$ ) substates. $g-i \otimes$ scan measured by XRD for as-grown vdWE BST film (g) and transferred BST membranes on flat glass $(\mathrm{h})$ and flexible PETE (i), respectively. 

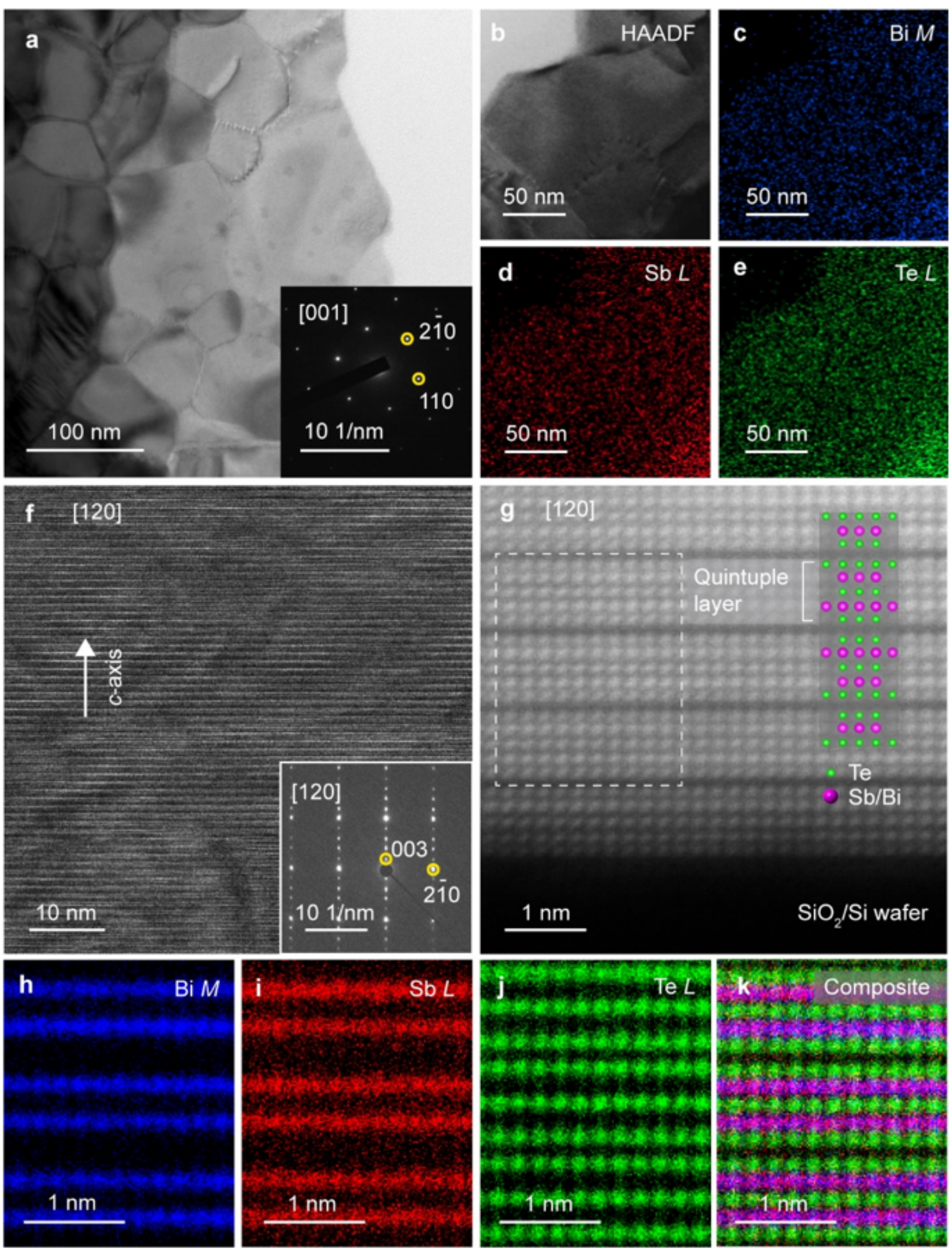

\section{Figure 3}

High-quality freestanding BST membrane. a Low magnification HAADF TEM image in the top view of exfoliated BST membrane. Inset of (a) shows the SAED pattern along [001] axis zone. b-e Low magnification TEM-EDS analysis of exfoliated BST film. $f$ HAADF TEM image in the cross-section view of transferred BST membrane. Inset of (f) shows the SAED pattern along [120] axis zone. g Atomic scale 
HAADF image of transferred BST membrane on the SiO2/Si wafer. $\mathrm{h}-\mathrm{k}$ Atomic scale TEM-EDS results of boxed region in $(\mathrm{g})$.
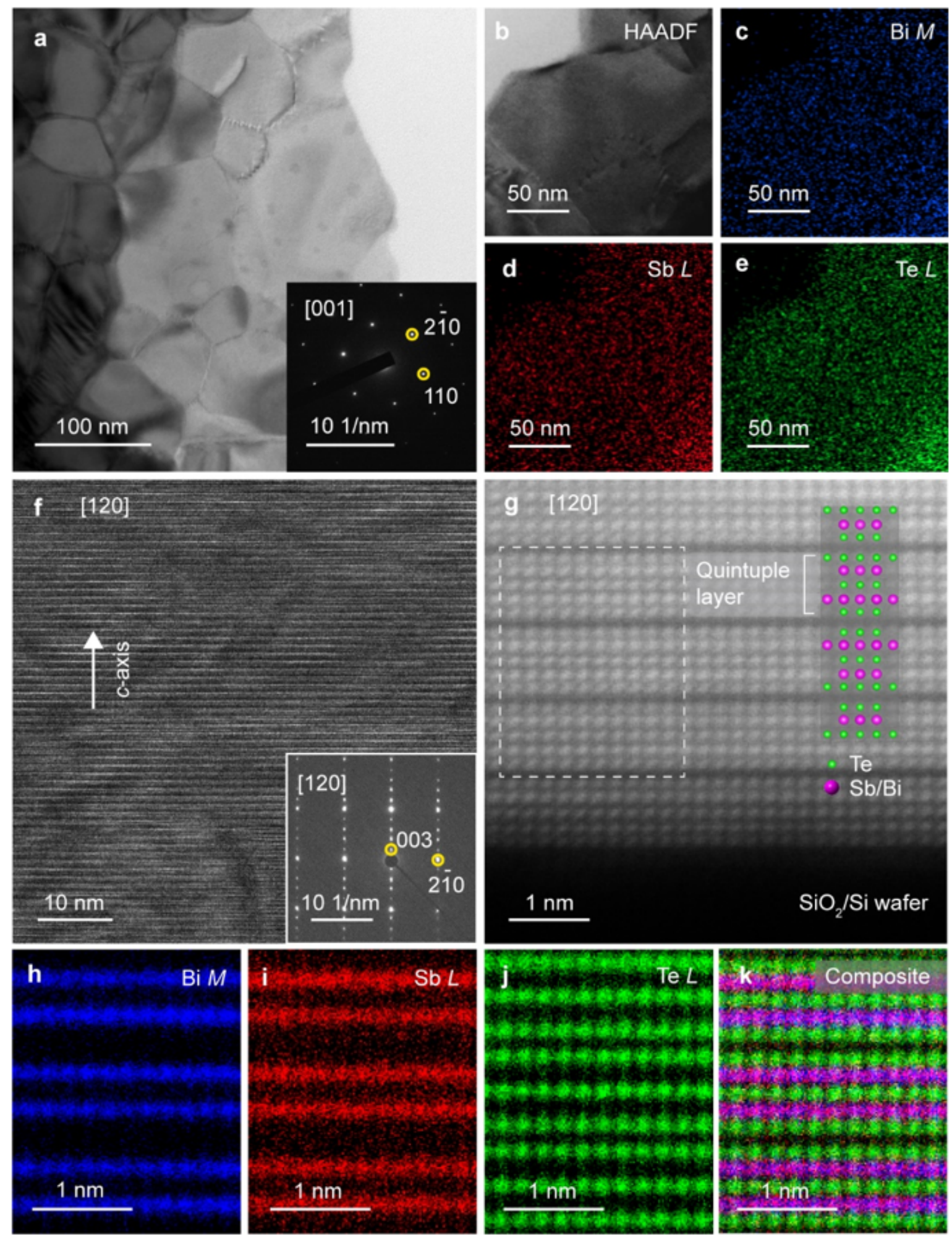

\section{Figure 3}

High-quality freestanding BST membrane. a Low magnification HAADF TEM image in the top view of exfoliated BST membrane. Inset of (a) shows the SAED pattern along [001] axis zone. b-e Low magnification TEM-EDS analysis of exfoliated BST film. $f$ HAADF TEM image in the cross-section view of 
transferred BST membrane. Inset of $(f)$ shows the SAED pattern along [120] axis zone. $g$ Atomic scale HAADF image of transferred BST membrane on the SiO2/Si wafer. $h-k$ Atomic scale TEM-EDS results of boxed region in $(\mathrm{g})$.

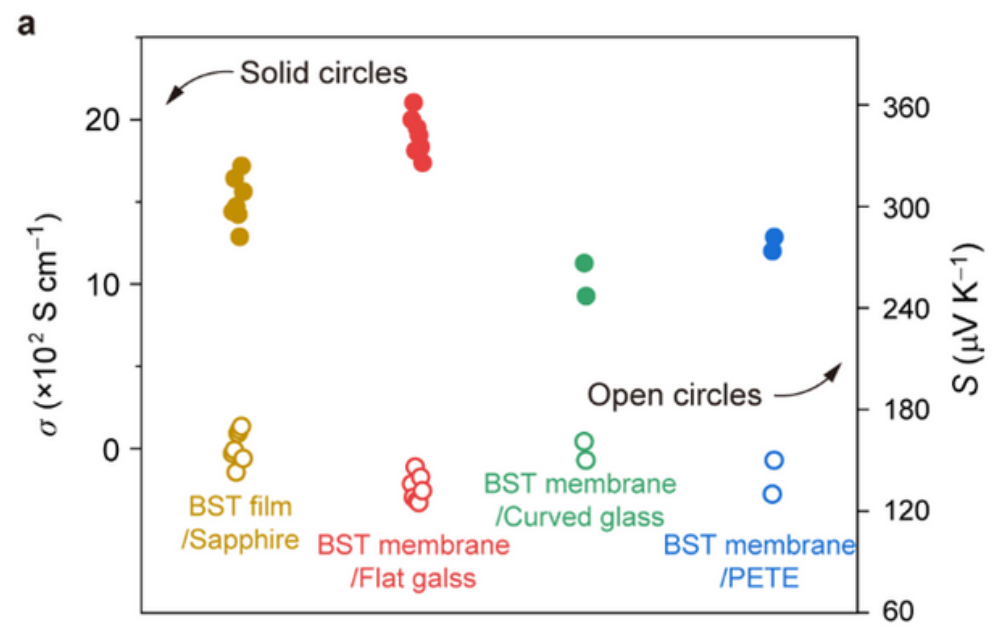

As-grown BST films and transfered BST membranes

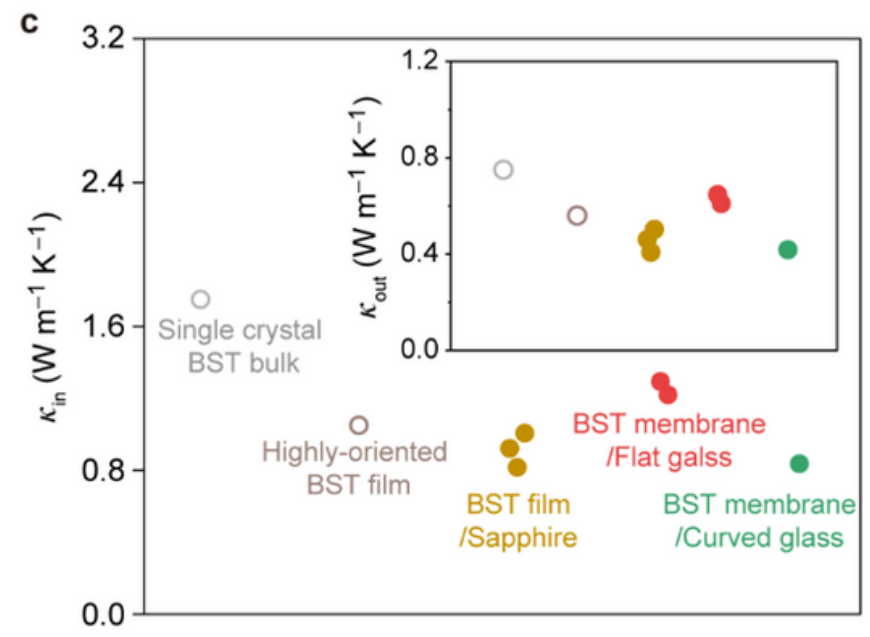

As-grown BST films and transfered BST membranes

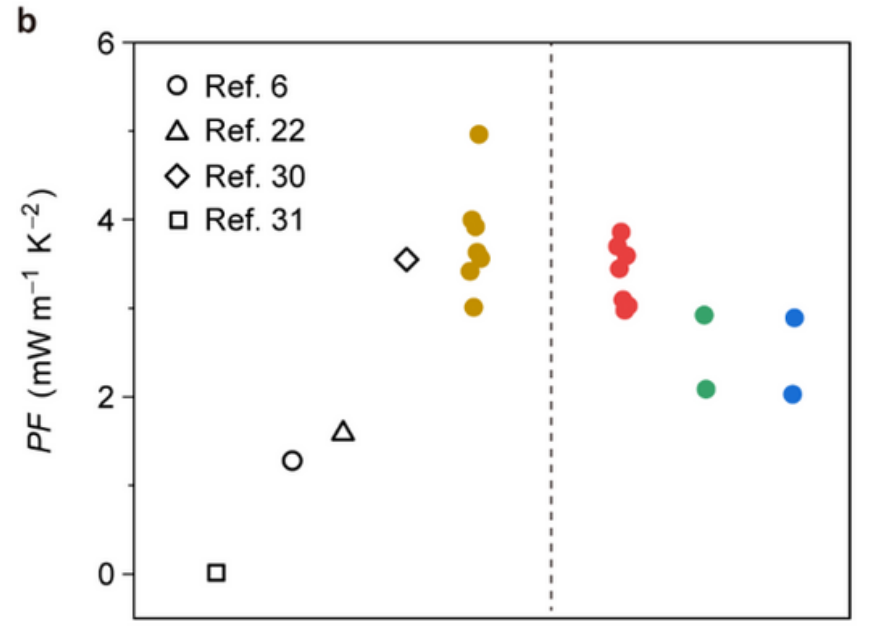

As-grown BST films

Transfered BST membranes

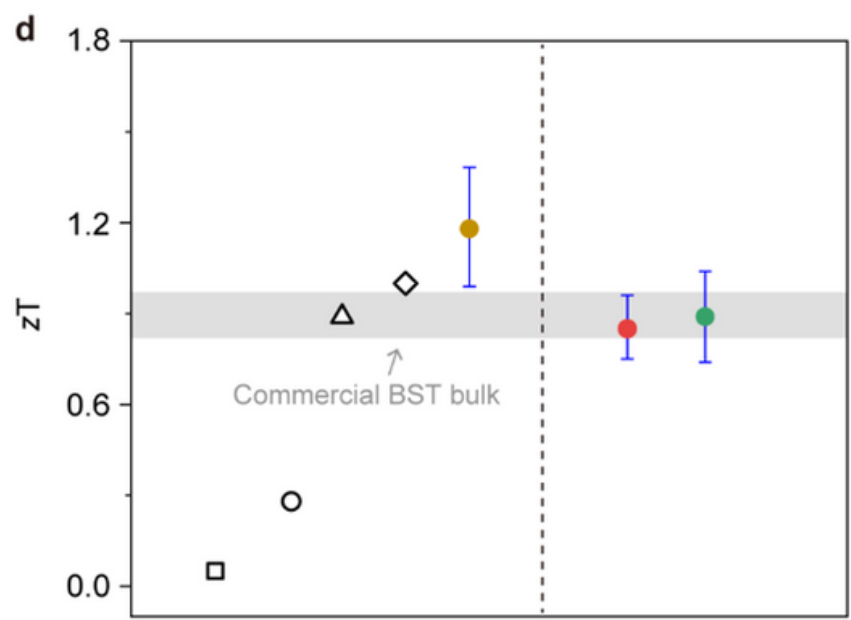

As-grown BST films
Transfered BST membranes

\section{Figure 4}

Thermoelectric properties of as-grown vdWE BST films and transferred BST membranes. $a-b$ Experimental in-plane electrical conductivity ( () ), Seebeck coefficient (S) and power factor (PF) of asgrown vdWE BST films and transferred BST membranes on flat glass, PETE and curved glass substrates at room temperature, respectively. c Estimated in-plane thermal conductivity of as-grown vdWE BST films and transferred BST membranes on flat glass and curved glass at room temperature. The reported inplane thermal conductivity values of single crystal BST bulk and highly-oriented nanocrystalline BST film with same composition are also shown for comparison. Inset in (c) shows the experimental out-of-plane thermal conductivity of as-grown vdWE BST films and transferred BST membranes on flat glass and curved glass. d Room-temperature zT values of as-grown vdWE BST films and transferred BST 
membranes on flat and curved glass. Reported zT values of several as-grown BST films are also shown for comparison.

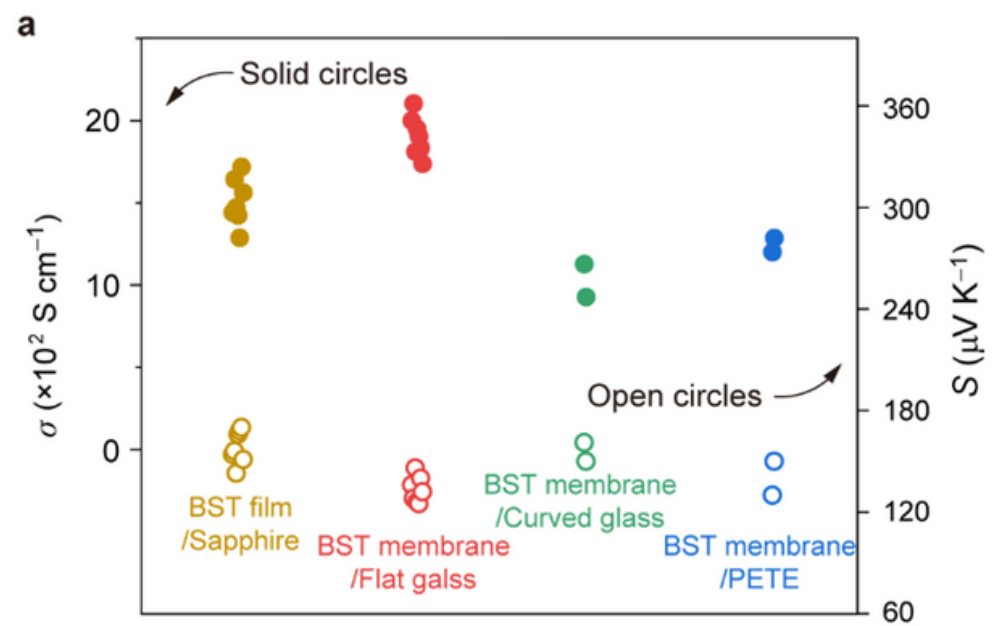

As-grown BST films and transfered BST membranes

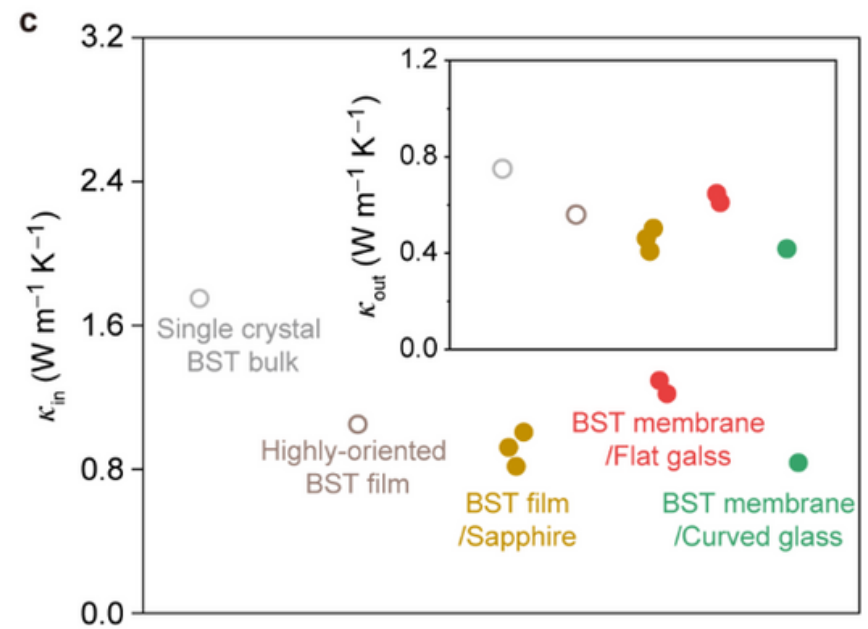

As-grown BST films and transfered BST membranes

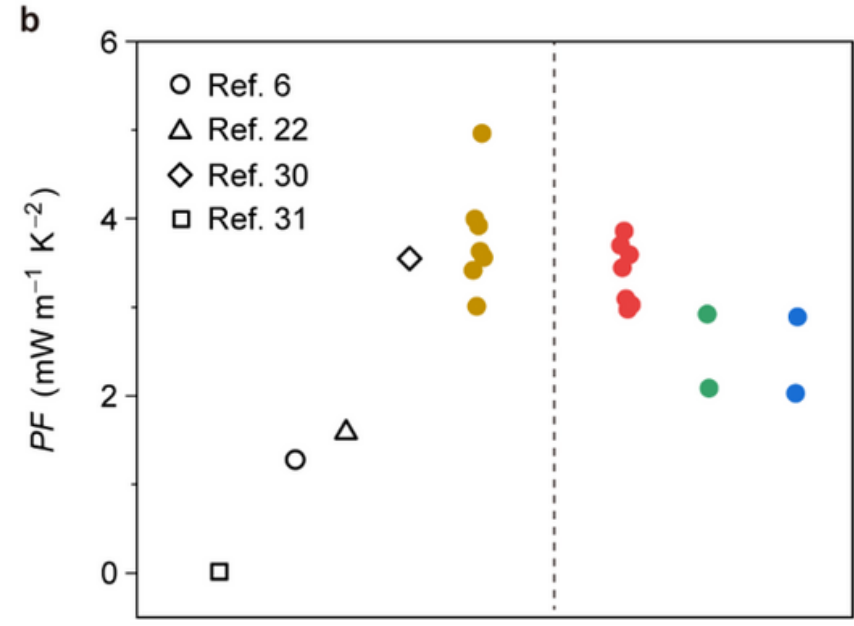

As-grown BST films

Transfered BST membranes

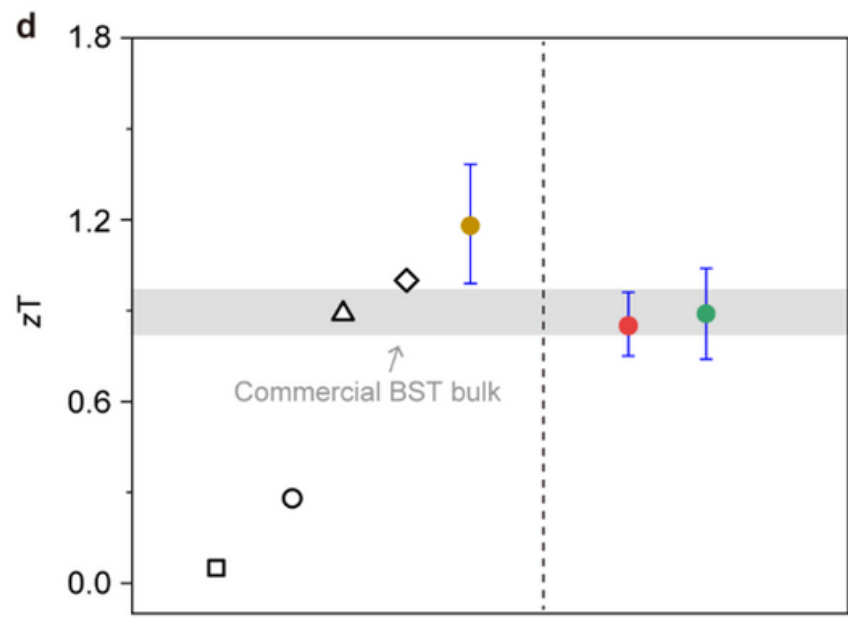

As-grown BST films
Transfered BST membranes

\section{Figure 4}

Thermoelectric properties of as-grown vdWE BST films and transferred BST membranes. $a-b$ Experimental in-plane electrical conductivity ( () , Seebeck coefficient (S) and power factor (PF) of asgrown vdWE BST films and transferred BST membranes on flat glass, PETE and curved glass substrates at room temperature, respectively. c Estimated in-plane thermal conductivity of as-grown vdWE BST films and transferred BST membranes on flat glass and curved glass at room temperature. The reported inplane thermal conductivity values of single crystal BST bulk and highly-oriented nanocrystalline BST film with same composition are also shown for comparison. Inset in (c) shows the experimental out-of-plane thermal conductivity of as-grown vdWE BST films and transferred BST membranes on flat glass and curved glass. d Room-temperature zT values of as-grown vdWE BST films and transferred BST membranes on flat and curved glass. Reported zT values of several as-grown BST films are also shown for comparison. 


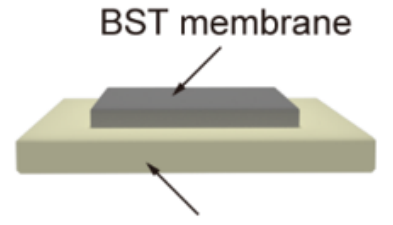

PETE
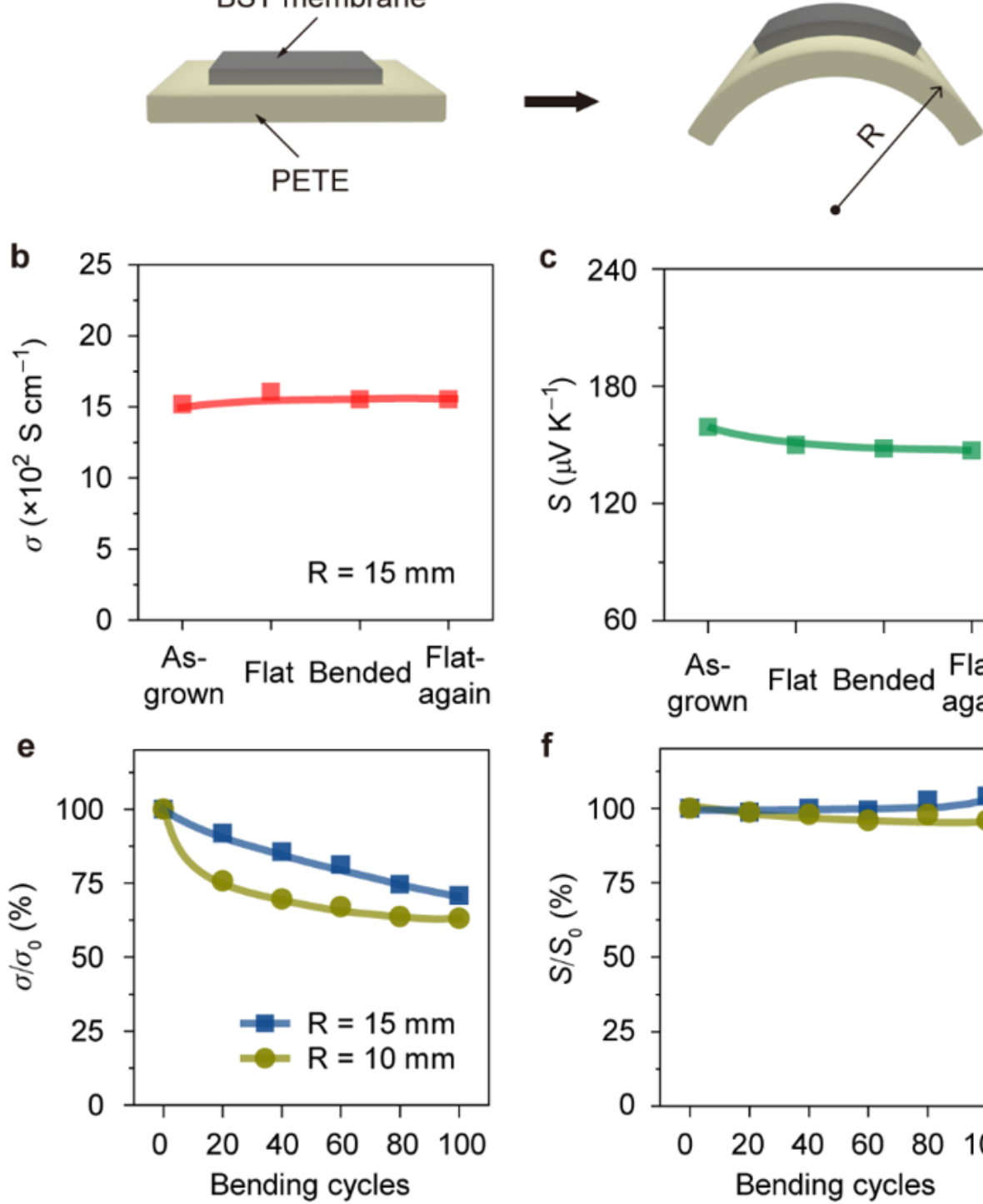

C

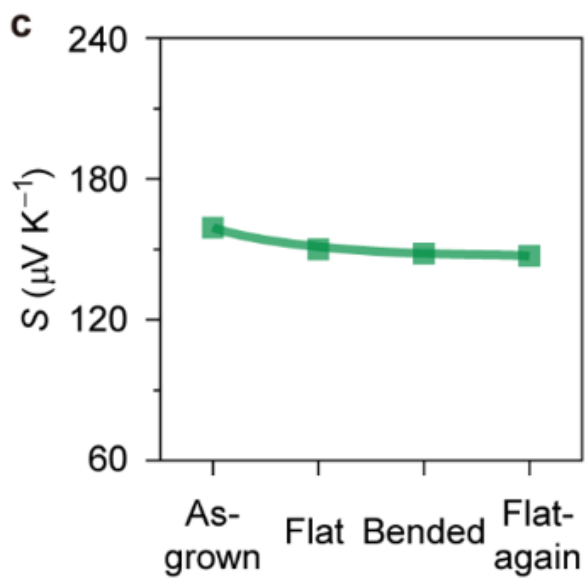

f

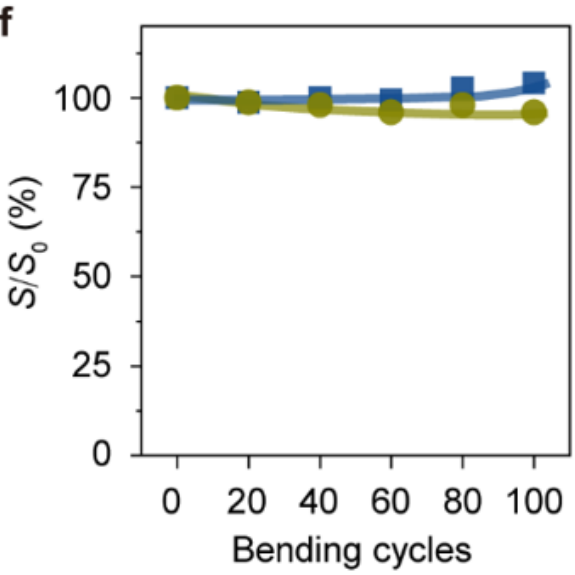

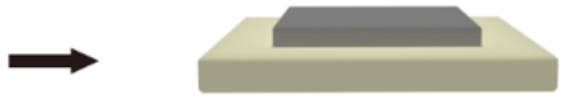

d

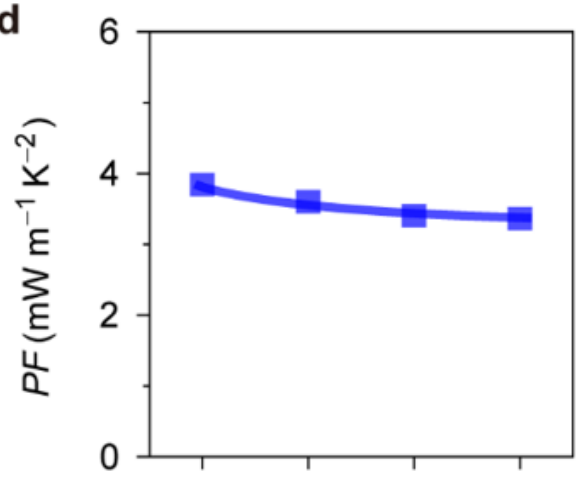

Asgrown

Flat Bended Flatagain

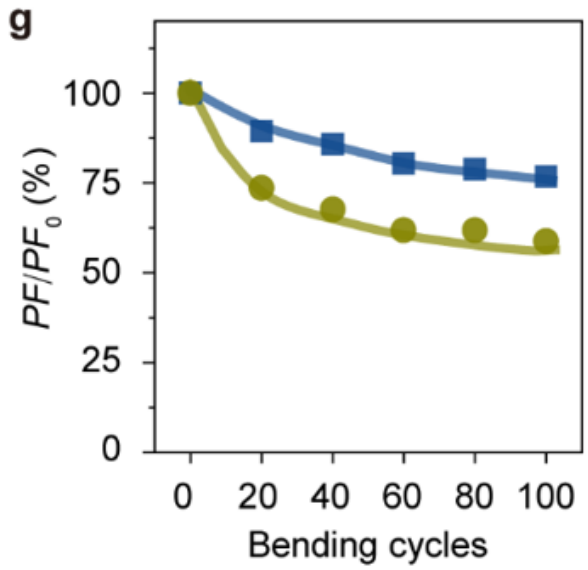

\section{Figure 5}

Bending test of freestanding BST membrane on flexible PETE. a Schematic illustration for a bending cycle of BST membrane on flexible PETE. b-d Electrical conductivity ( $\square$ ), Seebeck coefficient (S) and power factor (PF) of as-grown vdWE BST film and transferred BST membrane on PETE in one bending cycle. The bending radius is $15 \mathrm{~mm}$. e-g Relative electrical conductivity, Seebeck coefficient, and power factor of the BST membrane as a function of bending cycle. The two bending radii used are 10 and 15 $\mathrm{mm}$. The $₫ 0, \mathrm{~S} 0$ and PF0 are the values of the freestanding BST membrane when transferred on the flexible PETE. 

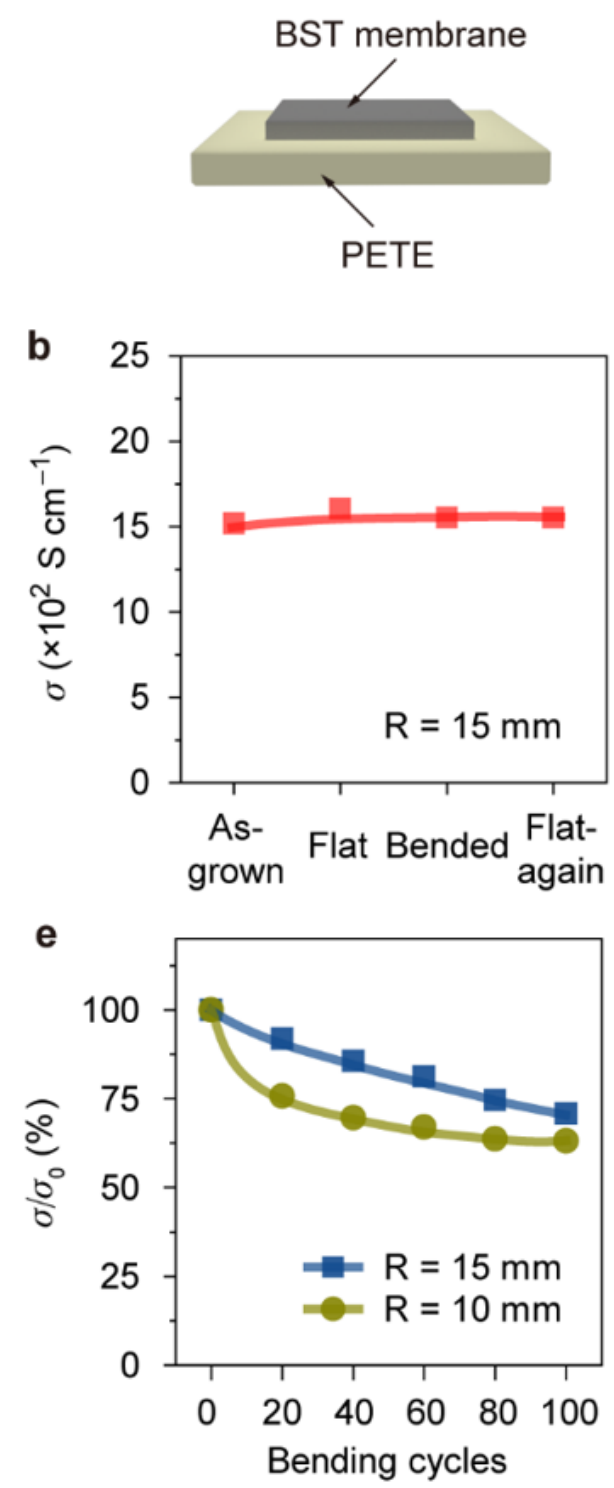
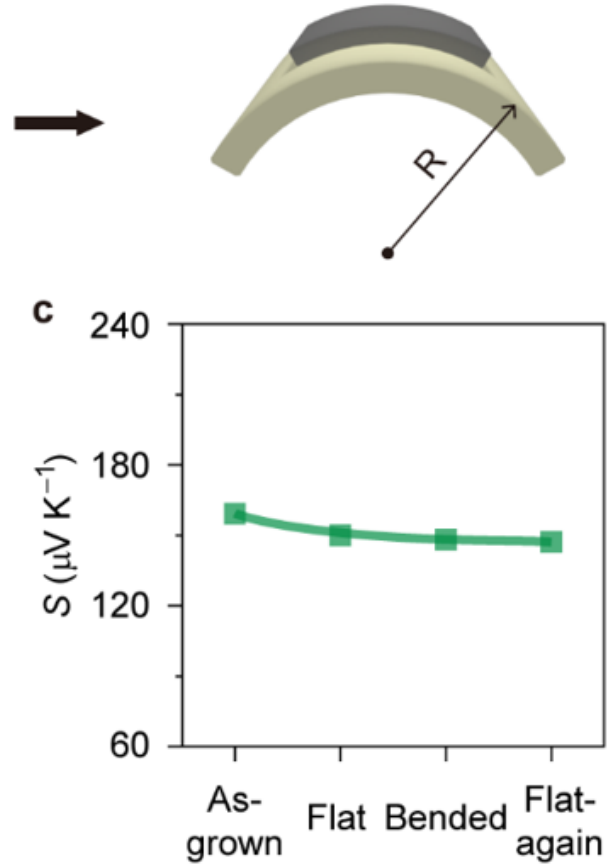

f

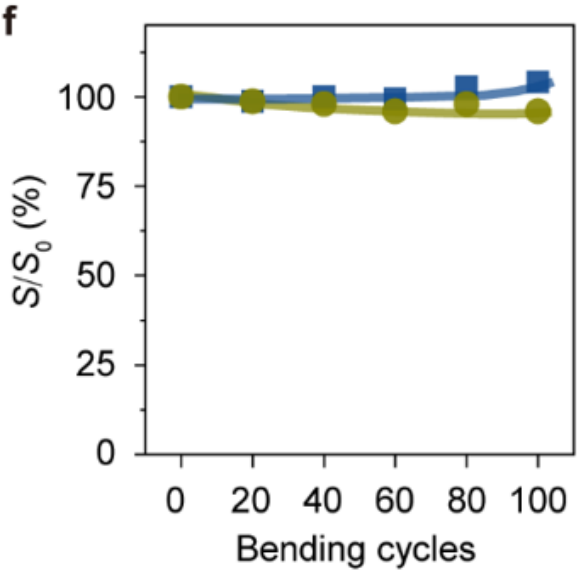

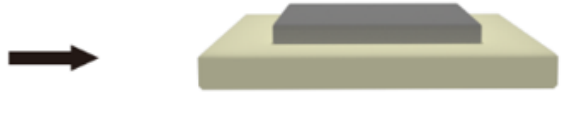

d

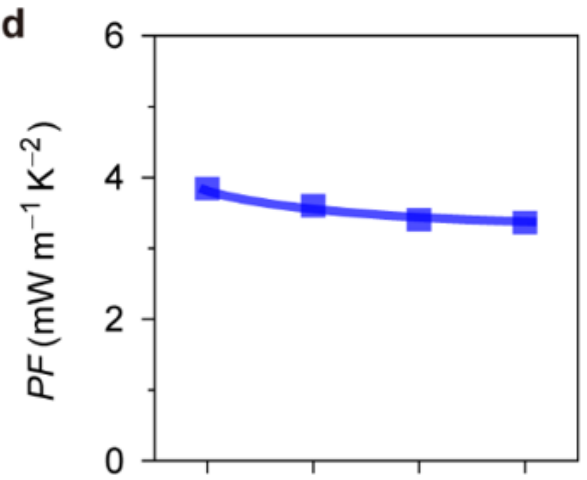

Asgrown

Flat Bended Flatagain

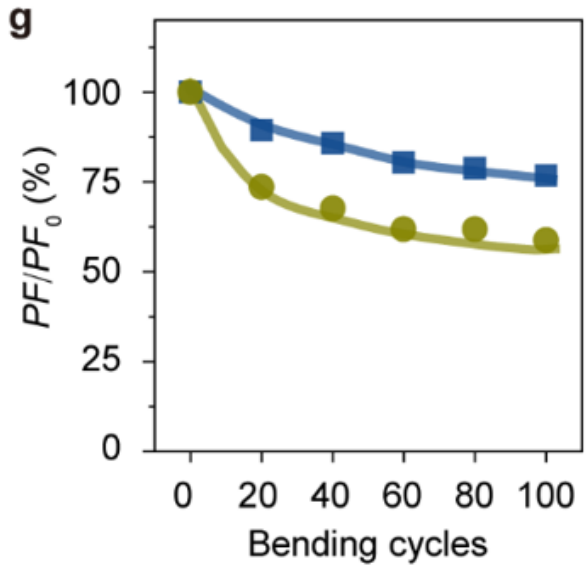

\section{Figure 5}

Bending test of freestanding BST membrane on flexible PETE. a Schematic illustration for a bending cycle of BST membrane on flexible PETE. b-d Electrical conductivity ( $($ )), Seebeck coefficient (S) and power factor (PF) of as-grown vdWE BST film and transferred BST membrane on PETE in one bending cycle. The bending radius is $15 \mathrm{~mm}$. e-g Relative electrical conductivity, Seebeck coefficient, and power factor of the BST membrane as a function of bending cycle. The two bending radii used are 10 and 15 $\mathrm{mm}$. The $\otimes 0, \mathrm{SO}$ and PF0 are the values of the freestanding BST membrane when transferred on the flexible PETE.

\section{Supplementary Files}

This is a list of supplementary files associated with this preprint. Click to download. 
- SuppleBSTTF.docx

- SuppleBSTTF.docx 